\title{
A chamber for separating visual and physical prey access from predators
}

\author{
CARL CHENEY and RON L. SNYDER \\ Institute of A nimal Behavior, Department of Psycholog!' \\ Utah State University, Logan. Utah 84322
}

\begin{abstract}
A chamber is described that provides automated separation of various phases of depredation dynamics. Two solenoid actuated doors control visual and physical access to prey items. A lever operandum is programmable to require differential response outputs by the predator. Search lever responses open one door and allow detection which may then be followed by pursuit lever responses and ultimate access for capture. The chamber is suitable for such medium sized predators as foxes and coyotes.
\end{abstract}

The investigation of predator-prey interaction demands the accessability of prey items to predators. A delivery system for providing such accessability in a laboratory situation has not been developed to any great extent. Dice (1947) released mice in a laboratory room with simulated natural cover and reported differential depredation by owls. Sparrowe (1972) tested sparrow hawks with a mouse "model" that moved back and forth between tunnels. Mouse killing by rats has received a great deal of attention from psychologists (e.g., Karli, 1956; Berg \& Baenninger, 1974), but these studies have not dealt comprehensively or operationally with the entire depredation episode. Ethologists and wildlife researchers ordinarily observe and report depredation activities as they occur in the organism's natural environment (e.g., Kaufman \& Wagner, 1973; Lawick \& Lawick-Goodall, 1970: Schaller, 1970) and therefore do not provide data of an analytical type.

The predator-prey interaction as a process can be divided into four basic phases: (1) search, (2) detection, (3) pursuit, and (4) capture. To study the process in the laboratory, we developed a chamber that allows. to a great extent, a simulation of these four phases. The chamber has been used with predators of the family Canidae and a variety of prey (rats, chicks, mice, guinea pigs, rabbits).

Figure 1 is a front view of the chamber indicating the overall dimensions. Three of these chambers are available to the predator on each trial. The basic chamber is a box constructed of 1/2-in. plywood with a solid floor and chickenwire roof for natural light. On the side of the front panel facing the predator is mounted a $10 \times 15 \mathrm{~cm}$ lever operandum (a), $20 \mathrm{~cm}$ from the ground and to the lower left of the access doors. (See Figure 1). Operation of this lever, which closes a microswitch, may be required in both search and pursuit phases. At least two

Research supported in part by University E \& M Grant V-58-41. Reprints may be obtained from either author. to five responses are required in order to avoid "accidental" operation of the lever as the predator searches about the chamber. All responses and door operations are recorded on a 6 -pen event recorder which, at a constant speed, provides interevent times, latencies, chamber cloice distributions, response patterns, etc.

Two doors are used to distinguish search from detection, and detection from pursuit. The outside door (b) is solid 3/4-in. plywood which, when closed, prevents physical and visual access to the chamber interior. Operating the lever (as an analog search activity) can result in this outside door falling open. For simplicity and reliable operation, the door is hung by the upper right corner on a pivot (c). Gravity allows the door to swing down and functionally out of the way (as shown

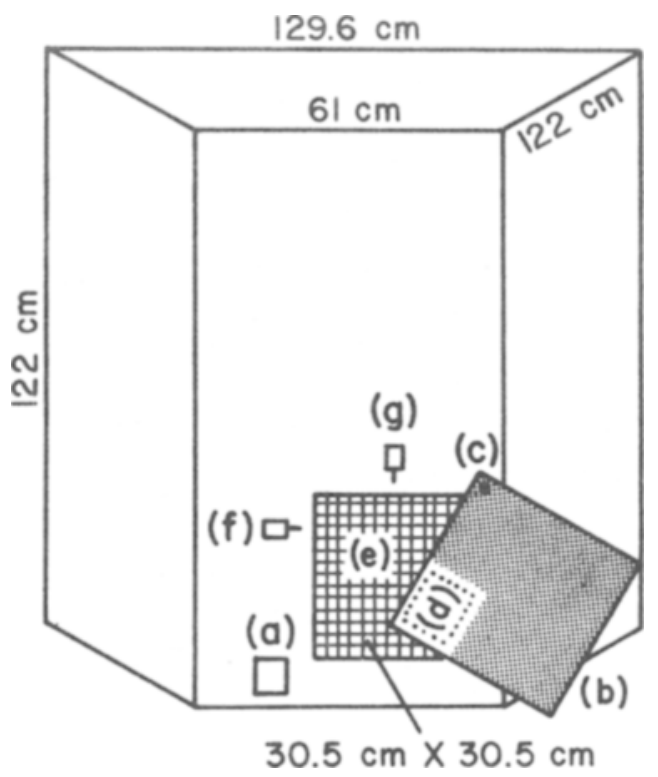

Figure 1. Schematic diagram showing the visual access door (b) open and the physical access door (e) closed. 


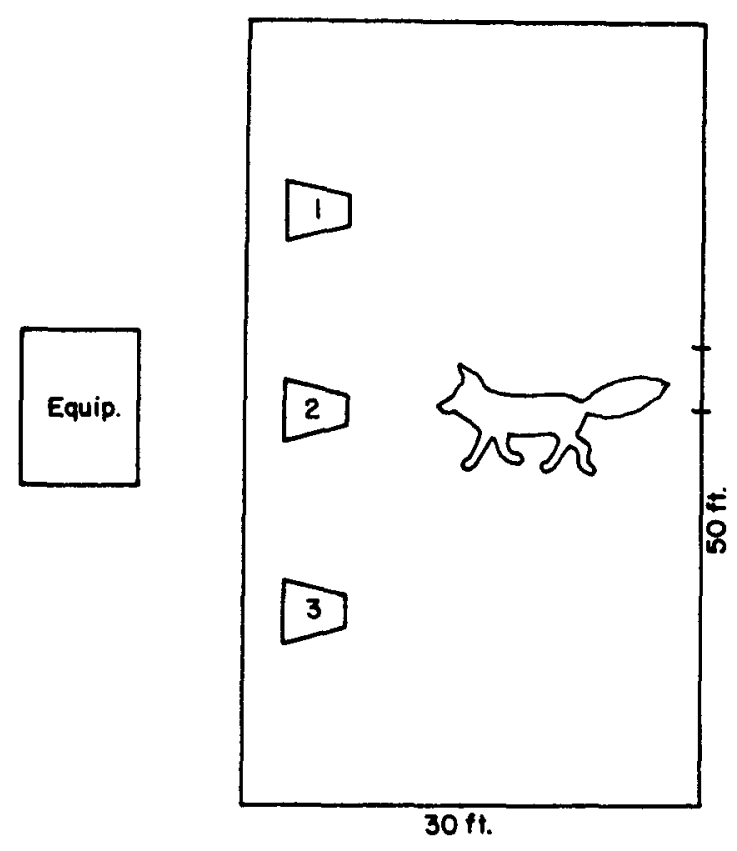

Figure 2. Predator-prey chambers in the study arena.

in Figure 1). A lead counterweight (d) in the upper left corner of the door aides in moving the door rapidly and further to the right. A magnet or dashpot device could be employed to dampen door oscillation, but, we have not found it to be necessary. This visual access door is held in the closed position and released by an ac solenoid (f). The inner door (e) is constructed of $1 / 2$-in. hardware cloth mounted in a frame, and hinged at the bottom (shown in the closed position in Figure 1). This door, when closed, prevents physical access to the interior but allows complete visual access. The chamber is roughly triangular in shape such that a freely moving prey item cannot easily move out of sight from a viewer located outside the visual access door. Door (e) is the physical access door in that operation of it's latching solenoid (g), and the subsequent expansion of a small rubber cushion located immediately behind $(\mathrm{g})$ and attached to the door, allows the door to fall in and down. A single $10-\mathrm{cm}$ long leg on the back of this door keeps it from falling completely to the floor, which might provide haven for a small prey animal. Operation of door (e) may also be controlled by a response requirement (pursuit) on the lever (a).

A description of the system in operation is as follows. The three chambers are each loaded with a prey item, and all doors are closed. A predator approaches a chamber and operates the lever by depressing it with a front paw a predetermined number of times (search). The visual access door (b) opens, and the predator "looks" in. If no prey item is detected, he moves to another chamber. If a prey item is detected (and presumably is desirable), pursuit may follow in the form of more responses on the lever until eventually the physical access door (e) opens. The predator may then hop inside the chamber for the capture. This fourth phase of the process can be recorded by a photocell system oriented midway across the door opening. Breaking the photobeam a second time (the first time indicates the access door opening) can be taken as an indication of capture and can provide additional information, such as prey catching efficiency. A further refinement at this stage is to close the door behind the predator trapping him inside the chamber. There may be some advantage to this procedure, and it could be automated by using a weighted cable connected to one of the doors and released as a function of photobeam interuption indicating entry by the predator.

Figure 2 illustrates one arrangement of the three chambers in our predator-prey arena. Interchamber distance can be manipulated; access may be allowed all around the chamber or to only the front panel, as well as other possible variations. The terminal steps (killing and consuming) are recorded by an observer, since these may occur anywhere in the arena. Eisenberg and Leyhausen (1972) have presented an extensive study of capture and killing behavior in mammals, but they were not concerned with the earlier phases of the predation episode.

Data have been collected using two species of fox (Vulpes macrotis and Vulpes fulva) involving over 2000 lever operations and 500 door openings. The simplicity of the system led to few problems, failures, or trial abortions. We have also used the chambers to compare prey selection probability on the basis of such factors as differential biomass, species, live vs dead, etc. The chambers are being used with coyotes, dogs, foxes, and felines. In addition to behavioral responses, physiological variables such as heartrate can be taken and correlated with all phases of depredation.

Snyder (1974) recently investigated probability matching. One of three chambers contained an accessible prey item per trial; prey items in the other two chambers (on a fixed probability basis) were retained in small cages which made them inaccessible to capture or consumption but did provide olfactory and auditory signals (visual signals were available with the visual access door open). The predators learned rapidly to abandon further effort on the lever (pursuit) when opening the visual access door revealed a caged, nonaccessible prey item. The third chamber contained a noncaged prey item. The purpose was to determine the manner in which the predator distributed its first-chamber choices over a long series of trials when one chamber contained an accessible prey item $10 \%$ of the time, Chamber 2 contained an accessible prey $30 \%$, and Chamber 3,60\% of the trials. 


\section{REFERENCES}

Berg, D. \& Baenninger, R. Predation: Separation of aggressive and hunger motivation by conditioned aversion. Journal of Comparative and Physiological Psychology, 1974, 86, 601-606.

Dice, L. R. Effectiveness of selection by owls of deer mice (Peromyscus maniculatus) which contrast in color with their background. Contributions from the Laboratory of Vertebrate Biology, University of Michigan, 1947, 34, 1-20.

Eisenberg, J. F., \& Leyhausen, P. The phylogenesis of predat ory behavior in mammals. Zeitschrift fur Tierpsychologie,. 1972 $30,59-93$.

Karli, P. The Norway rat's killing response to the white mouse; an experimental analy sis. Behavior, $1956,10,81-103$.

Kaufman, D. W., \& Wagner, C. K. Differential survival of white and agouti Mus musculus under natural conditions. Journal of Mamm ology, 1973, 54, 281-283.

Lawick, H. van, \& Lawick-Goodall, J. van. Innocent Killers, New York: Ballantine Books, 1970.

Schaller, G. B. The tiger and its prey. In, Field studies in natural history. New York: Van Nostrand Reinhold, 1970.

Snyder, Ron L. Probability matching in foxes. Paper presented at the annual Animal Behavior Society meeting, Urbana-Champaign, 1974.

Sparrowe, R. D. Prey-catehing in the sparrow hawk. Journal of Wild life Management, 1972, 36, 297-306.

(Received for publication September 24, 1974; revision accepted September 27, 1974.) 